\title{
Etude contributive à la connaissance des populations de simulies dans la commune de Bouaflé, Centre-Ouest de la Côte d'Ivoire
}

\author{
Grégoire YAPI YAPI*, François Dipomin TRAORE, \\ Drissa COULIBALY et Emmanuel TIA \\ Centre d'Entomologie Médicale et Vétérinaire, Université Alassane Ouattara de Bouaké, \\ Côte d'Ivoire, 27 BP 529 Abidjan 27, Côte d'Ivoire. \\ *Auteur correspondant: E-mail : yapigrec@yahoo.fr; Tél +225 224143 51/0837 72 78/40 376749 ;
}

Fax +22522415722

\section{RESUME}

Après l'arrêt des activités du programme de lutte contre l'onchocercose en Afrique de l'Ouest (OCP) en 2002, la Côte d'Ivoire n'a pu conduire régulièrement les activités de lutte du fait de la crise socio-politique qui a démarré en 2002. La recolonisation abondante des cours d'eau par les simulies et son corollaire la nuisance simulidienne le long de certains bassins versants, est due au fait que de vastes régions plus ou moins forestières n'ont jamais été traitées régulièrement par les insecticides contre les populations larvaires de simulies vectrices. L'objectif général du travail est de contribuer à la connaissance de la dynamique des populations de simulies et de la transmission de l'onchocercose en Côte d'Ivoire et particulièrement aux alentours du fleuve Marahoué dans la commune de Bouaflé. La technique de capture des simulies sur appât humain a été utilisée. Les résultats indiquent une forte nuisance simulidienne (117 piqûres / Homme / Jour) avec un pic l'après-midi. Les simulies capturées sont essentiellement $(99,1 \%)$ des espèces savanicoles ; elles présentent une grande longévité (64.44\%). Du point de vue transmission, aucune simulie femelle infectée ou infectieuse n'a été décelée pendant la durée de l'étude.

(c) 2014 International Formulae Group. All rights reserved.

Mots clés : Onchocercose, espèces savanicoles, dynamique de populations, nuisance simulidienne, transmission.

\section{INTRODUCTION}

L'onchocercose, connue encore sous le nom de «cécité des rivières », est une filariose cutanéo-dermique, débilitante et due à un parasite appelé Onchocerca volvulus. Elle est transmise par la piqûre des femelles desimulie (petite mouche noire) qui induit au point d'inoculation des lésions cutanées prurigineuses (Samba, 1995 ; Gentilini et al., 2012).
L'aire d'endémicité de l'onchocercose en Afrique se situe entre le $15^{\mathrm{e}}$ parallèle Nord et le $14^{\mathrm{e}}$ parallèle Sud (OMS 1994; OMS 1995). Consciente de l'ampleur épidémiologique et des répercussions socioéconomiques de cette maladie, l'Organisation Mondiale de la Santé (OMS) a mobilisé des moyens humains, matériels, techniques et financiers pour lutter contre l'onchocercose (Hougard et al., 2002). Ainsi, de 1974 à 1990, 
l'OMS a mis en place un vaste programme de lutte contre l'onchocercose dénommé OCP (Onchocerciasis Control Program) qui couvrait les pays endémiques de l'Afrique de l'Ouest. La stratégie de lutte autour de laquelle est axé le programme OCP est la lutte anti-vectorielle. Les rapports techniques ivoiriens sur l'onchocercose (IRD, 2001; IRIN, 2007 ; Anonyme, 2012 ; Banhoro, 2014) montrent que la lutte antivectorielle contre l'onchocercose a débuté en Côte d'Ivoire sur le bassin du haut Bandama, et initialement sur la haute Bagoé au milieu des années 1960 dans le cadre des campagnes FED-OCCGE ; elle s'est poursuivie jusqu'en 1974. Ces foyers ont été intégrés à partir de 1975 dans la première phase des opérations antivectorielles aériennes d'OCP. Les traitements ont été étendus aux hauts bassins des sous-affluents du Niger (haut Baoulé, haute Bagoé et affluents) à partir de 1977. Une extension des opérations (4 ${ }^{\text {ème }}$ phase opérationnelle d'OCP) a concerné à partir de 1979, le bassin du haut et moyen Sassandra, celui de la Marahoué, le bas Bandama en aval du lac de Kossou, le bassin inférieur du Nzi, la moyenne et basse Comoé environ jusqu'à Koutoukro. En 1990, les traitements larvicides étaient interrompus uniquement sur le bassin de la haute Comoé et la Volte Noire. Les derniers traitements concernaient le bassin du moyen Sassandra et ont été interrompus en 2001. Cette lutte a permis de rompre la transmission et d'éliminer la maladie en tant que problème de santé publique dans la majorité des pays affectés dont la Côte d'Ivoire (OMS/APOC, 2009 ;Sightsavers, 2011). L'OCP a arrêté ses activités en 2002 et a passé la relève au Programme africain de lutte contre l'onchocercose (APOC). En Côte d'ivoire, la lutte contre la maladie est pilotée par le Programme National de Santé Oculaire et Lutte contre l'Onchocercose (PNLSO-LO). Même si l'onchocercose maladie n'est plus une préoccupation pour la santé publique, la nuisance simulidienne est un cortège difficilement supportable dans certaines zones de la Côte d'Ivoire dont Bouaflé. C'est dans ce contexte de fortes densités agressives des simulies que nous avons mené sur quatre mois (Septembre à Novembre 2010 et en Janvier 2011) la présente étude le long du bassin de la Marahoué. La durée de l'étude s'inscrit dans la préparation d'un master en entomologie médicale et vétérinaire. De nombreux travaux (Garm, 1973; Bellec, 1976 ; Bellec et Hébrard, 1977 ; Phillipon, 1977 \& 1978; Quillivéré et al., 1977) ont déjà été consacrés au problème de populations de simulies (rythme journalier de piqûres, dynamique de populations: densité et âge moyen des femelles). L'objectif général du travail est de contribuer à la connaissance de la dynamique des populations de simulies et de la transmission de l'onchocercose en Côte d'Ivoire et particulièrement aux alentours du fleuve Marahoué dans la commune de Bouaflé.

\section{MATERIEL ET METHODES \\ Zone d'étude \\ Critères de choix de la zone d'étude}

Les critères de choix de notre zone d'étude étaient multiples:

- La commune de Bouaflé est située en zone forestière et bénéficie d'un important cours d'eau, la Marahoué ;

- Bouaflé fait partie des localités de la Côte d'Ivoire où l'on rencontre la majorité des maladies vectorielles telles que l'onchocercose;

- L'arrêt du traitement larvicide depuis 2002;

- L'existence d'une forte nuisance simulidienne avec une possibilité de réémergence de la maladie d'autant plus que depuis septembre 2002, la région est occupée par une importante population d'immigrés constitués en majeure partie de personnes (dont certaines sont probablement parasitées par Onchocerca volvulus) en provenance des 
zones contrôlées jadis par la rébellion.

\section{Considérations générales de la zone d'étude}

\section{Situation géographique}

Bouaflé est une ville située au Centre -

Ouest de la Côte d'Ivoire dans la région de la Marahoué entre $6^{\circ} 55^{\prime}-7^{\circ} 01^{\prime \prime}$ ' LN et $5^{\circ} 44^{\prime}-$ $5^{\circ} 49^{\prime} \mathrm{LW}$. Elle est située à $324 \mathrm{~km}$ d'Abidjan, capitale économique et à $58 \mathrm{~km}$ de Yamoussoukro, capitale politique de la Côte d'Ivoire. Elle fait frontière au Nord avec le département de Zuénoula, au Sud avec le département de Sinfra, à l'Est avec le district de Yamoussoukro et à l'Ouest avec le département de Daloa. Avec une population estimée à $236 \quad 312$ habitants selon le recensement de 1998, le département de Bouaflé s'étend sur une superficie de 4214,5 $\mathrm{km}^{2}$ (Figure 1).

Deux points de collectes des simulies ont été choisis pour notre étude. Le premier, Petit-Garango encore appelé Ousso-Yaokro est situé à environ $4 \mathrm{~km}$ du Centre-ville, sur l'axe Bouaflé-Daloa et le second, du nom d'Allangba-Konankro est à environ $5 \mathrm{~km}$ de la ville et situé sur l'axe Bouaflé-Sinfra.

\section{Climat}

Le département de Bouaflé appartenant au secteur préforestier, est caractérisé par deux saisons sèches et deux saisons pluvieuses. La saison sèche principale s'étant de novembre à février et la petite saison sèche, de juillet à Août. La grande saison des pluies va de mars à juin. De septembre à octobre, on a la petite saison des pluies. Les pluviométries annuelles enregistrées en 2002 et 2011 par la station ANADER de Bouaflé, sont respectivement $1226 \mathrm{~mm}$ et $1421 \mathrm{~mm}$ de pluie. La température moyenne oscille entre 26 et $27^{\circ} \mathrm{C}$ (Anonyme, 1999).

Relief

Le relief de la région de Bouaflé est constitué de plateaux et de plaines ; il est très peu accidenté, avec une altitude moyenne de
$250 \mathrm{~m}$. On y trouve cependant, quelques élévations dont le mont Lotanzia situé à 652 $\mathrm{m}$ de la chaîne Baoulé qui s'étend à l'Est de Bouaflé (Dibi et al., 2008).

\section{Végétation}

La végétation se présente comme une mosaïque de forêt semi-décidue et de savane. La savane dans cette région ressemble à une zone intermédiaire entre la savane herbeuse et les savanes arborée et arbustive (Dibi et al., 2008). Du fait des actions anthropiques, la structure originelle de la végétation a été fortement dégradée, laissant ainsi place à de grandes étendues de jachères et de plantations de cultures pérennes (Kouamé, 2008). La végétation riveraine (galeries forestières) le tapis des mousses sur les rochers, constituent le plus souvent au niveau d'accélération du courant, des gites préimaginaux de simulies (Bellec et Hébrard, 1977).

\section{Hydrographie}

Le réseau hydrographique se compose essentiellement du fleuve Marahoué ou Bandama rouge qui se divise en plusieurs bras, délimités par des affleurements du socle rocheux; leur nombre et positions varient selon les saisons (Bellec et Hébrard, 1977 ; Kouamé, 2008).

\section{Habitat et peuplement}

Peuplé de plus de 500 âmes, la population du site 1 (Petit-Garango se compose en grande partie de Baoulés mais également d'allochtones Malinkés et d'allogènes Burkinabés et Maliens. Dans cet habitat hétérogène, l'équipement en électricité et les structures socio-sanitaires sont absents. Le village bénéficie toutefois, d'un groupe scolaire public de douze classes, de puits et de deux forages équipés de pompes mécaniques. Peuplé de plus de 300 habitants, le second site d'étude (Allangba-Konankro) à la différence du premier, est exclusivement habité par les autochtones Baoulés et Gouros. Les étrangers y sont rares. Equipé d'un seul forage hydraulique, le village est dépourvu d'école 
primaire, d'électricité et de structures sanitaires.

\section{Activités économiques}

Les habitants des deux villages, à l'exception des Instituteurs, pratiquent essentiellement l'agriculture et/ou la pêche. L'igname, le manioc, la banane plantain, le riz pluvial, l'arachide et les légumes (gombo, piment, aubergine...) constituent les principales cultures vivrières auxquelles il faut ajouter le cacao et le café qui sont des cultures de rente. La pratique du commerce et de la mécanique font partie des activités recensées dans les sites d'étude.

\section{Méthodes}

\section{Capture des femelles de Simulies et dissection}

Les captures des simulies ont été effectuées sur appât humain de 7 heures à 18 heures (Quillivéré et al., 1977 ; Philippon, 1978, Eyong et al., 2006). Le captureur assis, jambes dénudées, récolte les simulies dans des tubes à hémolyse. IL prend la précaution de ne pas laisser le temps aux simulies de se gorger de sang. L'équipe de capture était composée de deux personnes qui assuraient la collecte des simulies, à tour de rôle pendant une heure. A la fin de chaque heure, l'agent inscrit sur une fiche de capture journalière, le nombre de femelles capturées et la température de l'air. Il indique également s'il a du vent ou la pluie. Les captures ont été effectuées pendant quatre mois, à raison de deux jours consécutifs par site, soit 4 jours / mois.

La dissection se fait sous une loupe binoculaire (Lewis, 1957, Le Berre, 1966, Philippon, 1978). Après l'analyse des ovaires, on sépare les femelles nullipares (femelles n'ayant jamais pondu) des femelles pares (femelles ayant effectué un ou plusieurs cycles gonotrophiques). Les femelles pares sont ensuite dilacérées dans une goutte d'eau physiologique, puis minutieusement examinées pour la recherche de larves d'Onchocerca volvulus. Le nombre, le stade d'évolution et la localisation de ces dernières ont été notés. Les simulies sont dites infectées lorsqu'elles hebergent des larves évolutives (L1, L2 ou L3), quelle que soit leur localisation. Les simulies hébergeant des larves infectantes dans la tête sont appelées simulies infectieuses (Enyong et al., 2005). Toutes les observations sont transcrites sur une fiche de dissection journalière qui est jointe à la fiche de capture.

\section{Identification des femelles de simulies}

Les différentes espèces du complexe Simulium damnosums ont identifiées grâce à la méthode d'identification morphologique des femelles (Quillivéré et al., 1977 ; Kurtak et al., 1981 ; Dang et Peterson, 1980 ; Garms et Zillmann, 1984; Baker et al., 1990; Wilson et al., 1993).

L'anlyse des résultats a permis de calculer les taux d'infestation, les charges parasitaires et l'intensité de transmission potentielle dans la zone. Dans un site donné, cette intensité est quantifiée par le potentiel de transmission qui est le nombre de larves infestantes morphologiquement indifférenciables d'O. volvulus hébergées par les simulies qui piqueraient un individu placé en permanence au point de capture (Duke, 1968). On calcule ainsi les potentiels mensuels de transmission (PMT) et la somme des potentiels mensuels de transmission mesurés sur une année constitue le potentiel annuel de transmission (PAT). 


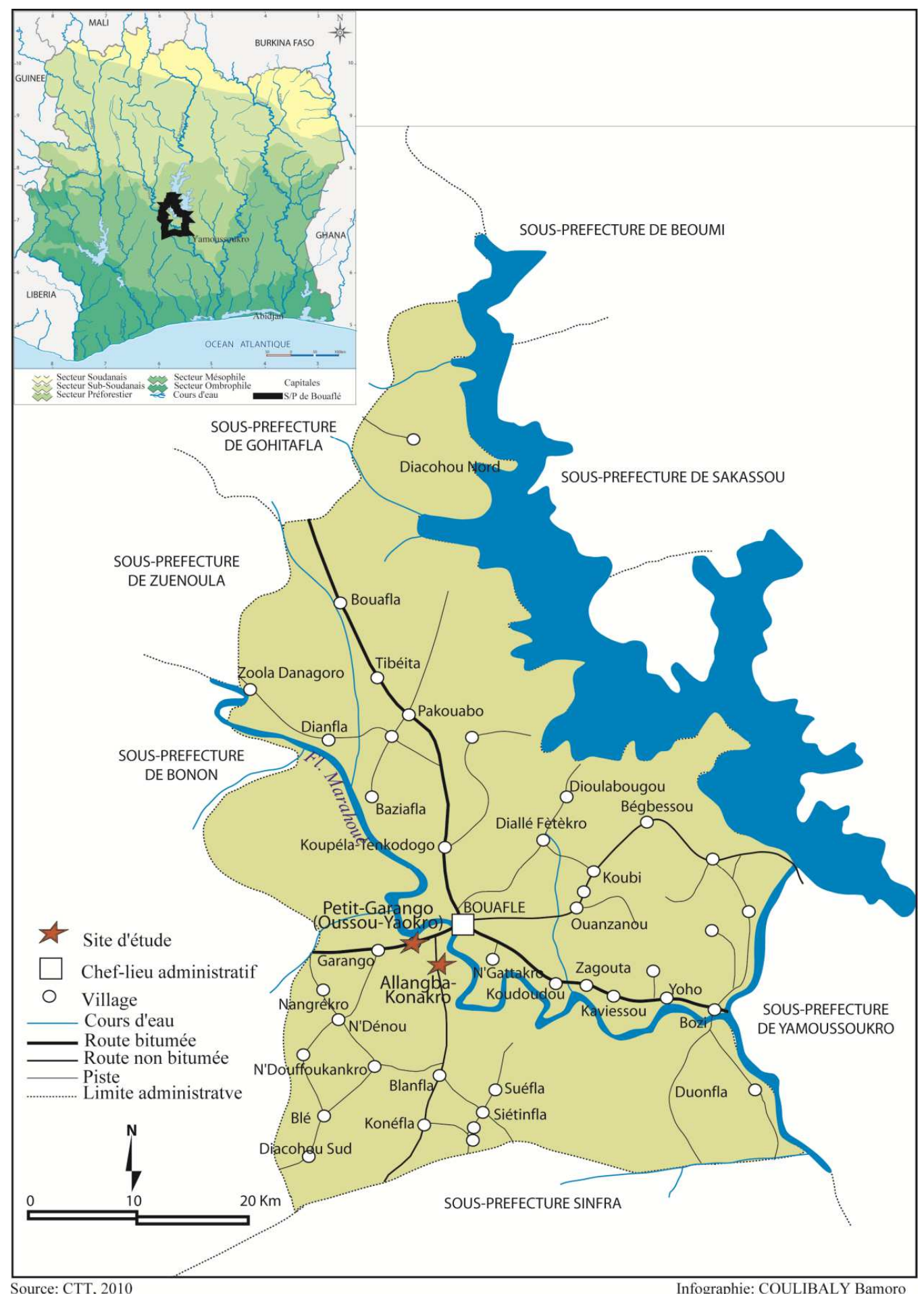

Figure 1 : Localisation des sites d'étude dans la commune de Bouaflé, Côte d'Ivoire. 


\section{RESULTATS}

Faune simulidienne

Deux groupes d'espèces de simulies pullulent le long du fleuve Marahoué: il s'agit des espèces du groupe de savane et de forêt. Le groupe savanicole comprend Simulium damnosum ss, $S$. sirbanum et celui de forêt, $S$. squamosum, $S$. sanctipauli, $S$. soubrense et $S$. yahense. Au total, 1862 simulies ont été capturées et 540 disséquées, soit un taux de 29\%. 99, 01\% (535/540) des simulies disséquées sont des espèces de savane et les simulies de forêt représentent $0,9 \%(5 / 540)$.

\section{Dynamique des populations de simulies}

\section{Taux d'agressivité journalière}

Le taux moyen d'agressivité journalière de la zone d'étude est de 117 piqûres/homme/jour $(\mathrm{p} / \mathrm{h} / \mathrm{j}$. Les simulies sont abondantes toute la journée sur les rives de la Marahoué. Elles sont plus agressives à partir de l'après-midi (Figure 2). Les variations horaires journalières de la nuisance simulidienne présentent deux pics dont l'un matinal et l'autre crépusculaire ou tardif. Le pic matinal s'obtient entre $9-10$ heures avec une moyenne de 46 piqûres/homme/heure. A partir de 14 heures, on assiste à une croissance exponentielle de cette agressivité qui culmine entre 17-18 heures (170 piqûres/homme/ heure).

Variations mensuelles des densités relatives de simulies

Au total, 1862 simulies ont été capturées en quatre mois d'étude (septembre à novembre 2010 et janvier 2011), soit un taux mensuel de piqûres (MBR) de 3510 piqûres. Le taux d'agressivité mensuel le plus élevé se situe en janvier (6 720 piqûres/homme/mois) et le plus bas, en novembre (380 piqûres/ homme/mois) (Tableau 1).

\section{Taux de parturité}

Sur 540 simulies femelles disséquées, 348 sont pares, soit un taux de parturité de $64,44 \%$ (Tableau 1). Les taux de parturité sont relativement élevés en saison des pluies (septembre-octobre). Ils sont plus faibles en saison sèche (novembre-janvier).

\section{Potentiel de transmission}

Durant notre étude, aucune simulie femelle n'a été retrouvée parasitée. Le potentiel mensuel de transmission (PMT) est donc nul pendant les quatre mois d'étude (Tableau 1).

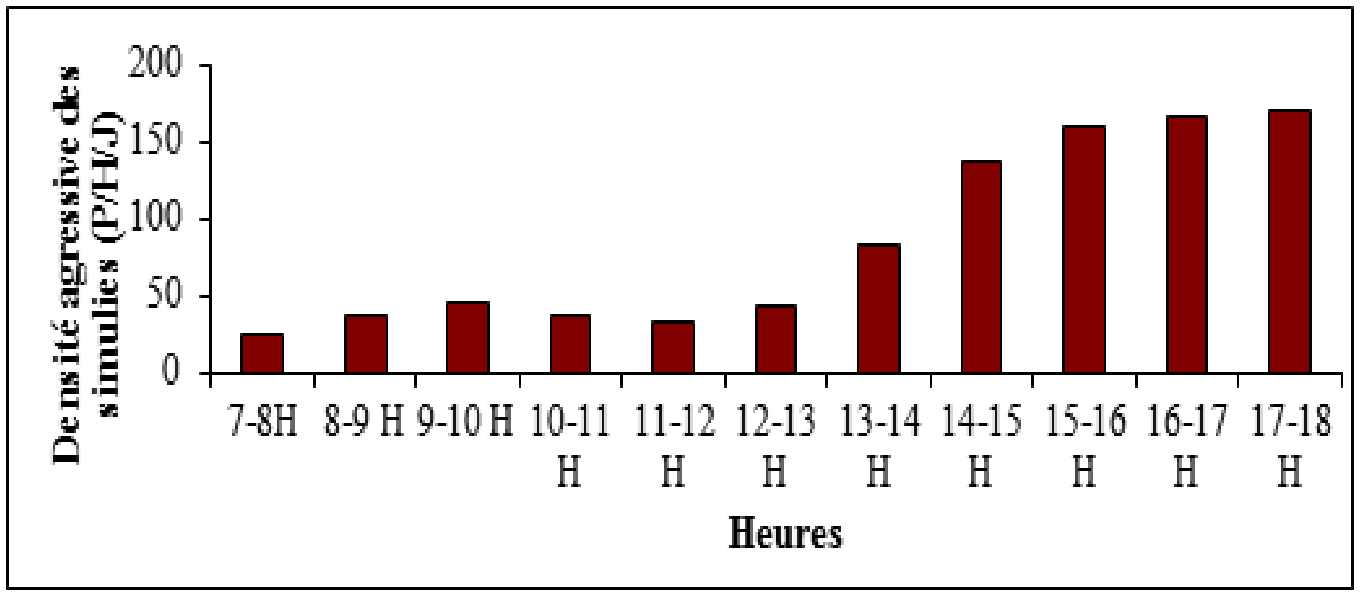

Figure 2: Variation journalière de l'agressivité des femelles de simulies dans la commune de Bouaflé (Côte d'Ivoire). 
Tableau 1: Paramètres entomologiques des simulies du fleuve Marahoué, dans la commune de Bouaflé, Côte d'Ivoire.

\begin{tabular}{lccccc}
\hline & \multicolumn{2}{c}{ Saison des pluies } & \multicolumn{2}{c}{ Saison sèche } & \multirow{2}{*}{ TOTAL } \\
\cline { 2 - 5 } & Septembre & Octobre & Novembre & Janvier & \\
\hline Nombre de jours de captures & 4 & 4 & 4 & 4 & $\mathbf{1 2}$ \\
Nombre de simulies capturées & 445 & 472 & 52 & 893 & $\mathbf{1 ~ 8 6 2}$ \\
Nombre de piqûres/homme/jour & 112 & 118 & 13 & 224 & $\mathbf{1 1 7}$ \\
Nombre de piqûres/homme/mois & 3360 & 3540 & 380 & 6720 & $\mathbf{3 5 1 0}$ \\
Nombre de femelles disséquées & 94 & 148 & 69 & 229 & $\mathbf{5 4 0}$ \\
Nombre de simulies savanicoles & 90 & 147 & 69 & 229 & $\mathbf{5 3 5}$ \\
Nombre de simulies forestières & 3 & 2 & 0 & 0 & $\mathbf{5}$ \\
Femelles pares & 71 & 120 & 38 & 119 & $\mathbf{3 4 8}$ \\
\% de femelles pares & 75,53 & 81,08 & 55,07 & 51,97 & $\mathbf{6 4 , 4 4}$ \\
Femelles infectées & 0 & 0 & 0 & 0 & $\mathbf{0}$ \\
\% Femelles infectées & 0 & 0 & 0 & 0 & $\mathbf{0}$ \\
Femelles infectieuses & 0 & 0 & 0 & 0 & $\mathbf{0}$ \\
$\%$ Femelles infectieuses & 0 & 0 & 0 & 0 & $\mathbf{0}$ \\
\hline Hauteur d'eau (cm) & 700 & 450 & 469 & 228 & $\mathbf{4 6 2}$ \\
\hline
\end{tabular}

\section{DISCUSSION}

\section{Dynamique des populations de simulies Faune simulidienne}

Les résultats de notre étude montrent que les espèces savanicoles constituent l'essentiel de la faune simulidienne capturée dans les deux sites. En effet, Bouaflé est une zone de transition entre la savane et la forêt. Avec la déforestation due à l'exploitation industrielle du bois et la création des plantations de culture de rentes, la forêt a presque disparue. Ainsi, les simulies du groupe savanicole venant du grand Nord du pays, envahissent de plus en plus cette région par le phénomène de migration, d'où la présence mixte des simulies forestières et savanicoles dans la zone d'étude. Il est établit que les mouvements de migration des simulies de savane vers les zones forestières sont liés à l'harmattan, vent saisonnier qui souffle du Nord vers le Sud. En cas de déforestation (cas de Bouaflé) et comme l'indique Philippon (1978), il peut également y avoir un simple phénomène d'occupation par extension de la zone devenue favorable aux espèces savanicoles.
Les rares espèces de simulies forestières sont capturées en saison des pluies. En cette période, le climat est frais, l'humidité relative atteint $85 \%$ et les arbres sont couverts de feuilles donnant l'allure d'une canopée le long de la forêt qui borde le fleuve Marahoué. Nos résultats concordent avec ceux de Barbazan et al. (1998), au Cameroun, qui ayant travaillé dans les mêmes faciès écologiques, ont rapporté que la saison des pluies est propice à la prolifération des simulies forestières. Le nombre de simulies de savane est élevé dans la zone d'étude et en cas de présence du parasite $O$. volvulus dans cet environnement, cela risque d'engendrer une recrudescence de la maladie. En effet, il est connu que le groupe de simulies de savane ( $S$. damnosumss $-S$. sirbanum) et Onchocerca volvulus de savane, forme l'un des couples les plus efficaces dans la transmission de l'onchocercose.

Variations journalières des densités relatives de simulies

Les rythmes journaliers d'activité des insectes hématophages sont étroitement liés aux variations de luminosité, de température et d'hygrométrie (Mavoungou et al., 2013). Le 
Berre (1966) a constaté que les simulies piquent uniquement le jour et que leur activité cesse lorsqu'il pleut ou que la température est trop élevée. D'autres auteurs (Giudicelli, 1966, Hausermann, 1969) ont indiqué que la température est le facteur limitant; l'hygrométrie n'a qu'une influence secondaire. A Bouaflé, Simulium damnosums L. est abondant le long des rives de la Marahoué dès le lever du jour jusqu'au coucher du soleil. La densité relative des simulies dans nos zones d'étude chute entre 11-13 heures, à cause de la forte chaleur observée à cette période. Cette abondance de simulies se traduit par un taux d'agressivité moyen relativement élevé de 117 piqûres/homme/jour pour la période d'étude. Ce nombre de piqûres reçues par homme/jour est largement suffisant pour déclencher les plaintes de la population à Bouaflé car le seuil tolérable est de 30 piqûres/homme/jour (Quillevéré, 1979). La nuisance simulidienne observée dans nos sites d'étude est comparable à celle rapportée par Doumdé (1985) qui est de 120 piqûres/homme/jour en région forestière de Côte d'Ivoire. Par contre, des densités supérieures aux nôtres ont été obtenues en d'autres lieux de la Côte d'Ivoire et notamment à Soubré (Pitroipa et al., 2002 ; Sylla, 2003) et à Palm-industrie Boubo (Coulibaly, 2006). Par ailleurs, les collectes de simulies réalisées sur piège ont permis à Bellec (1976), d'enregistrer 116 adultes/plaque/jour. Reste que dans ce dernier cas, il s'agit de l'ensemble de la population simulidienne, c'est-à-dire de toutes les catégories physiologiques confondues et non seulement des femelles à jeun qui sont proches physiologiquement des femelles piqueuses.

Variations mensuelles des densités relatives de simulies

Le fleuve Marahoué est un bassin versant très peu accidenté. Dans ce type d'habitat, les densités apparentes de simulies augmentent pendant les crues et baissent en période d'étiage (Le Berre, 1966).Outre la température qui influe significativement sur la densité apparente des simulies, la pluviométrie et son corollaire le niveau du cours d'eau jouent également un rôle régulateur de la dynamique des populations simulidiennes. Quand les gîtes sont noyés (hautes eaux), les densités apparentes chutent. Par contre en période de basses eaux, les densités relatives de simulies sont élevées à cause de l'existence de nombreux supports végétaux et granitiques immergés. Il n'y a pas uniformité partout, car Barbazan et al. (1998) lors d'une étude au Cameroun, ont montré que les densités simulidiennes augmentent pendant les périodes de crue et diminuent en période d'étiage.

Selon l'OCP, il est admis que lorsque le taux annuel de piqûre (TAP) est inférieur à 1 000 piqûres/homme/an dans une zone donnée, cette zone est favorable aux activités humaines. Dans nos zones d'étude, le cumul des TMP (3 510 piqûres) des quatre mois est largement supérieur à celui préconisé par l'OCP. Ces forts taux de TMP enregistrés mettent en exergue les problèmes réels de nuisance simulidienne que subissent les populations riveraines de la Marahoué.

\section{Dynamique de la transmission de l'onchocercose}

\section{Taux de femelles pares}

Le taux de parturité moyen est de $64,44 \%$. La population simulidienne est vieille dans la zone d'étude. Cette grande longévité est liée à la forte présence d'espèces savanicoles qui vivent généralement longtemps.

En effet, l'âge physiologique moyen des simulies de savane est plus élevé que celui des simulies forestières. Il se situe autour de $60 \%$ pour les espèces de savane et oscille entre 20 et $50 \%$ pour les espèces de forêt (Philippon, 1978). Les conditions écologiques n'étant pas favorables dans la savane, les simulies se déplacent moins (risque réduit) et consomment donc peu d'énergie. Ce comportement augmente par conséquent leur longévité. 
Le taux de parturité est relativement élevé en petite saison des pluies (septembreoctobre) et en grande saison sèche (janvier). La petite saison sèche qui vient juste avant la petite saison des pluies est courte, elle dure deux mois (juillet- août). Cette saison n'offrant pas les conditions de vie favorables, les simulies se déplacent très peu. Cet état de sédentarité relative augmente leur durée de vie et leur permet d'atteindre la petite saison pluvieuse. De même, les mauvaises conditions écologiques pourraient expliquer en partie le taux élevé de femelles pares obtenu en janvier.

\section{Potentiel mensuel de transmission.}

Le potentiel mensuel de transmission (PMT) est nul à Bouaflé pour chacun des 4 mois d'étude. Cela pourrait s'expliquer par le faible nombre de personnes infectées, d'où l'absence de simulies infectées rencontrées dans nos sites d'étude. En effet, l'âge physiologique relativement élevé (64\%) indique que les simulies prennent régulièrement leur repas de sang dans la zone. C'est dire que si ce sang hébergeait des microfilaires d'Onchocerca volvulus, cela se traduirait par un nombre plus ou moins grand de simulies portant le parasite.

\section{Conclusion}

L'étude longitudinale sur la dynamique de population de simulies conduite de septembre à novembre 2010 et janvier 2011 dans la commune de Bouaflé, Centre-Ouest de la Côte d'Ivoire, montre que la quasitotalité des simulies rencontrées sont des espèces savanicoles. L'âge physiologique élevé de ces simulies leur permet d'avoir plusieurs cycles gonotrophiques et entretenir facilement le cycle du parasite. Mais malgré leur âge physiologique élevé, nous n'avons trouvé aucune simulie infectée par Onchocerca volvulus pendant la durée de l'étude.

Bien que les résultats obtenus ne couvrent pas une année de recherche, les données nous permettent d'affirmer que l'onchocercose maladie ne représente pas encore un problème de santé publique dans la zone d'étude. Toutefois, la forte densité simulidienne constituée en majorité d'espèces savanicoles plus réputée dans la transmission de l'onchocercose nécessite une surveillance du programme national de lutte contre l'onchocercose.

Nous comptons, dans un proche avenir, conduire dans d'autres villages riverains de la Marahoué une étude épidémiologique prenant en compte l'évaluation parasitologique chez l'homme en complément de l'étude entomologique sur une année et une étude génétique pour élucider la dynamique populationnelle des simulies, la dynamique de transmission et l'existence d'espèces hybrides issues des simulies de savane et de forêt dans cette zone de transition forêt-savane.

La courte durée de notre étude ne permet de tirer que des conclusions partielles. Or la déforestation s'accentue et les populations deviennent plus nombreuses. C'est dire qu'il s'avère nécessaire de mener une étude couplée à une étude entomologique étalée sur un an. Au terme de ces travaux, il sera alors possible de conclure si les modifications du milieu ont eu un impact sur le comportement des vecteurs, entraînant un plus grand risque pour la population.

\section{REMERCIEMENTS}

Ce travail a été réalisé avec l'appui financier du Centre d'Entomologie Médicale et vétérinaire dans le cadre de la formation des étudiants inscrits en Master 2 d'entomologie médicale.

\section{REFERENCES}

ANADER. 2002. Rapport d'activités préliminaires des enquêtes techniques menées sur le terrain dans le Département de Toumodi. Service Technique ANADER, Toumodi, p.4.

ANADER. 2011. Rapport d'activités préliminaires des enquêtes techniques menées sur le terrain dans le Département de Bouaflé. Service Technique ANADER, Bouaflé, p.4. 
Anonyme. 1999. Biodiversité biologique de la Côte d'Ivoire (projet de synthèse). Ed. Ministère de l'Environnement et de la forêt, 19-31.

Anonyme. 2012. Lutte contre l'onchocercose/ La campagne de sensibilisation et d'information démarre aujourd'hui : voici les districts sanitaires concernés. Dossier Santé, journal le Mandat, p.1.

Banhoro S. 2014. L'onchocercose: les partenaires relancent la lutte. Frat_mat.info, p.1.

Baker RHA, Guillet $P$, Sékétéli A, Poudiougou P, Boakye D, Wilson MD, Bissan Y. 1990. Progress in controlling the reinvasion of windborne vectors into the western area of the Onchocerciasis Control Programme in West Africa. Philosophical Trans. Roy. Soc. London, B328: 731-750.

Barbazan P, Escaffre H,Mbentengam R, Boussinesq M. 1998. Etude entomologique sur la transmission de l'onchocercose dans une zone de transition forêt-savane du Cameroun. Bull. Soc. Pathol. Exot., 91(2): 178-182.

Bellec C. 1976. Captures d'adultes de Simulium damnosumTheobald, 1903 (Diptera, Simulidae) à l'aide de plaques d'aluminium, en Afrique de l'Ouest. Cah ORSTOM, série Ent. Med. Parasitol., 16(3): 209-217.

Bellec C, Hébrard G. 1977. Captures d'adultes de simulidae, en particulier de Simulium damnosum Theobald 1903, à l'aide de pièges d'interception: les pièges-vitres. Cah ORSTOM, série Ent. Med. Parasitol., 15(1): 41-54.

Boakey DA. 1993. Apictorial guide to the chromosomal identification of members of the Simulium damnosumTheobld Complex in West Africa with particular reference to the Onchocersiasis control Programme area. Trop. Med. Pathol., 44: 223-244.

Coulibaly F. 2007 Evaluation de l'efficacité de la crème à base d'huile de palmiste (Elaeis guineensis) dans la lutte contre la nuisance simulidienne sur le site agroindustriel de la Palmci-Boubo (Divo, Côte d'Ivoire). Mémoire de DEA (CEMV), Université Alassane Ouattara, p.66.

Dang PT, Peterson BV. 1980. Pictorial keys to the main species and species groups within the Simulium damnosum Theobald complex occurring in West Africa (Diptera: Simuliidae). Trop. Parasitol., 31 : 117-120.

Dibi NH, Yao C, Adou Y, N'guessan, Koné M, Yao SC. 2008. Analyse de la diversité floristique du parc National de la Marahoué, Centre-Ouest de la Côte d'Ivoire. Afrique Science, 3: 552-579.

Doumdé N. 1985 Evaluation entomologique de la lutte contre Simulium damnosums L. en Côte d'Ivoire. Relation avec les phénomènes de résistance aux insecticides et de ré-invasion des zones protégées du programme de lutte contre l'onchocercose dans le bassin de la volta (O.C.P.). Mémoire de DEA (CEMV), Université de Cocody, Abidjan, p.42

Duke B. 1968. Studies of factors influencing the transmission of onchocerciasis. IVThe biting cycles, infective biting density and transmission potential of "forest" Simuliumdamnosum. Ann. Trop. Med. Parasitol., 62: 95-106.

Enyong P, Traoré S, Demanou M, Esum M, Fobi G, Noma M, Kyembé D, Sékétéli A. 2005.Programme Africain de lutte Contre l'Onchocercose (APOC): intensité de la transmission d'Onchocerca volvulus par Simulium squamosum dans deux régions de la République du Cameroun. Entomologie médicale. Bull. Soc. Pathol. Exot., 99(4): 272-277.

Enyong P, Traoré S, Louango E, Fobi G, Noma M, Yaméogo L, Kyembé D, Sékétéli A. 2006. Programme Africain de lutte Contre l'Onchocercose (APOC): transmission de Onchocerca volvulus par Simulium damnosums.l. dans deux régions de la République Centrafricaine. Parasite, 13: 35-44. 
Garms R. 1973. Quantitative studies on the Tranmission of Onchocerca volvulus by Smilium damnosum in the Bong range, Liberia. Tropenmed Parasit, 24: 358-372.

Garms R, Zillmann U. 1984. Morphological identification of Simulium sanctipauli and S.yahensein Liberia and comparison of results with those of enzyme electrophoresis. Trop. Parasitol., 35: 217220.

Gentilini M, Danis M, Richard-Lenoble D, Caumes E, Begué P, Touze JE. 2012. Médecine Tropicale (6 $6^{\mathrm{ème}}$ edn). Médecine Sciences, Lavoisier : Paris ; 333-347.

Giudicelli J. 1966. Récoltes de simulies en Côte d'Ivoire. Etude de l'activité diurne des femelles de Simulium damnosum Theobald. Ann. Soc. Ent. Fr. (NS), 11(2): 325-342.

Hausermann W. 1969. On the biology of Simulium damnosum Theobald, 1903, the main vector of Onchocerciasis in the Mahenge Mountains, Ulanga, Tanzania. ActaTropica, 26(1): 29-69.

Hougard JM. 1998. Blackfly control: What choices after onchocerciasis? World Health Forum, 19(3): 281-284.

Hougard JM, Yaméogo L, Philippon B. 2012. Onchocerciasis in West Africa after 2002: a challenge to take up. Parasite, 9(2): 105-111.

IRD. 2001. Vingt ans de lutte contre l'onchocercose : bilan écologiques. Rapport Technique, IRD, p.2.

IRIN. 2007. Côte d'Ivoire : recrudescence de l'onchocercose dans les zones forestières. Conférences pour l'onchocercose, Bruxelles, 19/09/07, p.2.

Kouamé SAK. 2008. Mise en place et entretien des productions végétales et / ou animales: cas du cacao. Mémoire d'ingénieur. Institut National Polytechnique Felix Houphouet Boigny, Yamoussoukro, p.65.

Kurtak DC, Raybould JN, VajimeCG. 1981. Wing tuft colours in the progeny of single individuals of Simulium damnosum
(Enderlein). Trans. Roy. Soc. Trop. Med. Hyg., 75: 126.

Le Berre R. 1966. Contribution à l'étude biologique et écologique de Simulium damnosum Theobald 1903 (DipteraSmuliidae). Mémoire O.R.S.T.O.M., 17: 204 p.

Lewis DJ. 1957. Aspects of the structure, biology and study of Simulium damnosum. Ann. Trop. Med. Parasitol., 51: 340-358.

Mavoungou JF, Acapovi-Yao GL, Kohagne L, Tongue, Zinga Koumba RC, MbangNguema OA, Obame- Ondo PK, M'Batchi B, Gilles J, Duvallet G. 2013. Influence du degre de perturbation du milieu sur l'activité journalière des stomoxys spp. (Diptera:Muscidae) au Nord-Est du Gabon. Revue CAMES, 1: 54-60.

OMS. 1994. Vingt années de lutte contre l'onchocercose en Afrique de l'Ouest. Bilan des activités du programme de lutte contre l'onchocercose en Afrique de l'Ouest de 1974 à 1994. OMS/OCP, 178 $\mathrm{P}$.

OMS. 1995. L'onchocercose et la lutte antionchocerquienne. Rapport d'un comité OMS d'experts de lutte antionchocerquienne. Genève, 113 p.

OMS. 2007. Lutte contre l'onchocercose dans la région africaine: situation actuelle et perspectives. Rapport du Directeur régional à la cinquante-septième session Brazzaville, République du Congo, 6 p.

OMS/APOC. 2009. Rapport de la $29^{\text {ème }}$ session du Comité Consultatif Technique (CCT), Ouagadougou. Dir/Coord/APOC/ Rap/CCT 29, p.115.

Philippon B. 1977 Etude de la transmission d'Onchocerca volvulus (Leuckart 1893) (Nematoda, Onchocercidea) par Simulim damnosum Theobald 1903 (Diptera, Simulidea) en Afrique tropicale. Thèse de Doctorat es-Sciences Naturelles d'Etat, Paris, p.308. 
Philippon B. 1978. L'onchocercose humaine en Afrique de l'Ouest. Initiative ORSTOM, Paris, 38: p. 193.

Pitroipa X, Sankara D, Konan L, Sylla M, Doannio JMC, Traoré S. 2002. Evaluation de l'efficacité de l'huile de coco contre les piqûres de Simulium damnosums L. Med. Trop. 62(5): 511516.

Quillivéré D. 1979. Contribution à l'étude des caractéristiques taxonomiques, bioécologiques et vectrices des membres complexe Simulium damnosum présents en Côte d'Ivoire. Travaux et documents de l'ORSTOM, 109, p.304.

Quillivéré D, Sechan Y, Pendriez B. 1977. Etude du complexe Similium damnosum en Afrique de l'Ouest VII. Etude de la bioécologie et du pouvoir vecteur des femelles de $S$. sanctipauli, S. soubrense et S. yahense en côte d'Ivoire. Cah ORSTOM, sér. Ent. Méd. Parasit., 15(4) : 301-329.

Samba EM. 1995. Le programme de lutte contre l'onchocercose en Afrique de l'Ouest : un exemple de bonne gestion de la santé publique. OMS, Genève, 107p.

Sightsavers. 2011. Elimination de l'Onchocercose : plan d'action accéléré dans 24 pays recevant le soutien de Sightsavers 2011-2021: $N^{\circ} 207544$ et Sc 038 110, p. 69.

Sylla M. 2002. Evaluation de l'efficacité de différentes formulations de répulsifs à base d'huile végétale contre les piqûres de Simulium damnosum en côte d'ivoire. Mémoire de DEA d'entomologie médicale et vétérinaire, Université de Bouaké, p.68.

Traoré S. 1997. Contribution à l'étude du comportement et des caractéristiques vectrices naturelles des espèces forestières $\mathrm{du}$ complexe Simulium damnosum en Côte d'Ivoire. Thèse de Doctorat d'Etat es sciences Naturelles, Université de Cocody, Abidjan, p.298.

Traoré S. 2008 Onchocercose et Simulies. Cours Magistral, Centre d'entomologie Médicale et Vétérinaire (CEMV), Université de Bouaké, p.20.

Wilson MD, Post RJ, Gomulski LM. 1993. Multivariate morphotaxonomy in the identification of adult females of the Simulium damnosumTheobald complex (Diptera: Simuliidae) in the Onchocerciasis Control Programme area of West Africa. Ann. Trop. Med. Parasitol., 87: 65-82. 